\title{
Enfisema y tabaquismo en pacientes con exposición laboral al amianto
}

\author{
Emphysema and smoking in patients \\ with occupational exposure to asbestos
}

\author{
David Macía Suárez¹, Estela Sánchez Rodríguez², María José Bello Peón ${ }^{3}$, Carmen Diego ${ }^{3}$ \\ ${ }^{1}$ Hospital Universitario Lucus Augusti. ${ }^{2}$ Universidade de Vigo. ${ }^{3}$ Complexo Hospitalario Universitario de Ferrol
}

\begin{abstract}
Objective: To study the association between the smoking degree and the appearance of emphysema in patients with a history of asbestos exposure in our region.

Material and methods: Patients included in the screening program of asbestos exposure are reviewed, including both tobacco exposure and thoracic CT studies of patients valuated between June 2012 and August 2013, recording both their status as non-smokers, former and current smokers, as well as their accumulated smoking rate (when applicable). Patients were classified according to the presence of paraseptal, centrolobulillar or diffuse emphysema in imaging studies.

Results: A total of 55 patients were included. A statistically significant difference $(p=0.006)$ was found at cumulative smoking data in number of patients with paraseptal emphysema compared to patients without emphysema (55.60 \pm 28.25 packages/year versus $28.19 \pm 23.25$ packages/ year).

Conclusions: Tobacco consumers exposed to asbestos present more often paraseptal emphysema than those non-smokers exposed to asbestos.
\end{abstract}

Keywords: Asbestos; Tobacco; Pulmonary emphysema

\section{Introducción}

Se conoce desde hace tiempo que el tabaquismo induce, a través de múltiples mecanismos, la aparición de patología, tanto pulmonar como a otros niveles ${ }^{1}$, incluyendo el desarroIlo de enfisema mediante un desequilibrio enzimático entre proteasas y antiproteasas ${ }^{2}$. De igual modo, es sabido que la exposición a fibras de asbesto condiciona, tras largos períodos de latencia, la aparición de patología a múltiples niveles, no exclusivamente respiratorios ${ }^{3}$. Con posterioridad ha sido demostrada la relación entre ambos factores ${ }^{4}$, en una relación más que sumatoria que ha llegado a ser descrita como sinérgica ${ }^{5}$ por algunos autores.

Resulta difícil realizar estudios en este tipo de pacientes, debido a que la fisiopatología determina elevados períodos de latencia (incluso superiores a 30 años ${ }^{3}$ ), implicando problemas de seguimiento a largo plazo de una cohorte de pacientes. En nuestra región la exposición al amianto ha sido elevada en determinados entornos laborales, como el sector de la construcción naval. Por ello, desde el año 2006, se realiza en

\section{Resumen}

Objetivo: Estudiar la asociación entre el grado de tabaquismo y la aparición de enfisema en pacientes con antecedentes de exposición al asbesto en nuestra región.

Material y métodos: Se estudian los datos de exposición al tabaco y se revisan los estudios de TC torácica de los pacientes incluidos en el protocolo de revisión por exposición laboral previa al amianto realizados entre junio de 2012 y agosto de 2013, registrando tanto su condición de no fumadores, ex-fumadores o fumadores, como su grado de tabaquismo acumulado (cuando corresponda), así como clasificando a los pacientes en función de la aparición de datos de enfisema (paraseptal, centrolobulillar o difuso) en los estudios de imagen.

Resultados: Se incluyeron un total de 55 pacientes. Se demostró una diferencia estadísticamente significativa $(p=0,006)$ al comparar los datos de tabaquismo acumulado en número de paquetes/año para los pacientes que presentaban enfisema paraseptal respecto a los que no, siendo la media de paquetes/año de $55,60( \pm 28,25)$ paquetes/año frente a 28,19 $\pm 23,25$ paquetes/año.

Conclusiones: Los pacientes consumidores de tabaco expuestos al amianto presentan con mayor frecuencia enfisema paraseptal que aquellos pacientes no fumadores expuestos al amianto.

Palabras clave: Asbesto; Tabaco; Enfisema pulmonar

colaboración con Atención Primaria un protocolo de vigilancia en los ex-trabajadores expuestos.

Se realizó una búsqueda bibliográfica en los principales buscadores (PubMed, Embase, Google scholar) no encontrando ningún estudio que abordase la asociación entre el grado de tabaquismo y la aparición de enfisema en pacientes con antecedentes de exposición al asbesto en nuestro país. Conocer dicha asociación constituyó el objetivo del presente trabajo.

\section{Material y métodos}

El presente trabajo se trata de un estudio observacional. El período de reclutamiento fue desde junio de 2012 hasta agosto de 2013 (14 meses). Los pacientes incluidos fueron seleccionados del protocolo de vigilancia en los ex-trabajadores expuestos a fibras de amianto que se realiza por parte de Atención Primaria desde el año 2006 en nuestra Área Sanitaria. Este protocolo, de carácter voluntario, incluye la realización anual de una anamnesis, con historia clínica y exploración física específica, así como la obtención de estudios de radiología simple y espirométricos. Cuando al- 
guno de ellos muestra alguna alteración, los pacientes son remitidos a la consulta específica de Neumología en donde se evalúan solicitando un estudio de Tomografía computarizada (TC). A todos aquellos pacientes enviados para su estudio específico, se les ofertó la posibilidad de formar parte del estudio, constituyendo el criterio de inclusión en el trabajo. La no asistencia a la consulta posterior a la realización del estudio de imagen y el rechazo a la participación en el trabajo fueron considerados los únicos criterios de exclusión, dado que no había menores de edad en el protocolo de vigilancia. Una vez obtenida autorización, se realizó un estudio de TC torácica, sin contraste intravenoso, con el paciente en decúbito supino y realizando una apnea sostenida en inspiración forzada, para la que previamente había sido entrenado por el personal técnico responsable, siendo distribuidos de manera aleatoria entre una de estas dos máquinas:

- TC multidetector de 64 cortes (LightSpeed VCT ST, General Electric Healthcare, Milwaukee, WI, USA).

- TC multidetector de 16 cortes (BrightSpeed, General Electric Healthcare, Milwaukee, WI, USA).

En la máquina de 64 detectores el protocolo aplicado fue el siguiente:

Tipo de exploración helicoidal, con un tiempo de rotación de 0,6 segundos, cobertura de detector $40 \mathrm{~mm}$, espesor helicoidal 0,625 mm, pitch 0,984:1, velocidad $39,37 \mathrm{~mm} /$ rot, SFOV large body, kV 100, mA 350 con smart mA, con algoritmo de reconstrucción de imagen "Soft" $(W=350, L=40)$, realizando reconstrucciones retrospectivas complementarias con el algoritmo "Lung" (W=1500, L=-700) y "Soft" (W=400, $L=40$ ), ambas con intervalos y espesores de $0,625 \mathrm{~mm}$, los mínimos que permite la máquina.

En la máquina de 16 detectores debieron realizarse algunos ajustes por motivos técnicos, quedando el protocolo de la siguiente manera:

Tipo de exploración helicoidal, con un tiempo de rotación de 0,6 segundos, 16 filas de detector con cobertura de $20 \mathrm{~mm}$, espesor de corte de $1,25 \mathrm{~mm}$, pitch 1,375:1, velocidad 27,5 $\mathrm{mm} / \mathrm{rot}$, SFOV large, a $120 \mathrm{kV}$ y $225 \mathrm{~mA}$, con tipo de reconstrucción "Stnd" ( $W=350, L=40)$, añadiendo reconstrucción retrospectiva con el algoritmo "Lung" (W=1500, $L=-500)$ y con algoritmo "Soft" ( $W=400, L=40)$, ambas con espesores de $1,25 \mathrm{~mm}$, intervalos de $0,800 \mathrm{~mm}$, los mínimos que permite la máquina.

Los estudios fueron sistemáticamente visualizados en las consolas de trabajo de los equipos (Advantage Workstation GE Healthcare Volume Share 3, con versión de software AW version vxtl_8_43), por un radiólogo con más de seis años de experiencia en este tipo de pacientes, con plena libertad para modificar los parámetros de visualización y postprocesado de imágenes, hasta un grosor de corte máximo de cinco milímetros, en reconstrucción multiplanar en función de las necesidades o dudas diagnósticas, incluyendo una valoración en algoritmo de TC de alta resolución (con un grosor de corte milimétrico).
Se establecieron dos grupos de pacientes, aquellos que presentaban datos de enfisema y aquellos que no los presentaban, clasificando a los primeros en:

- Enfisema paraseptal: Presencia de zonas transparentes sin engrosamientos septales asociados, de predominio subpleural, frecuentemente con bullas subpleurales añadidas, que traduce dilataciones de conductos y sacos alveolares ${ }^{6}$ (Figura 1).

- Enfisema centrolobulillar: Áreas multifocales transparentes sin paredes visibles que afectan preferentemente a los bronquiolos respiratorios del centro de los acinos y lobulillos secundarios. Es más frecuente en fumadores y lóbulos superiores 6 .

- Enfisema difuso: Reducción uniforme de la atenuación parenquimatosa y disminución de las estructuras vasculares. El enfisema centrolobulillar severo puede ser indistinguible ${ }^{6}$.

Para mantener ciego al revisor y proteger los datos de los pacientes, todos ellos fueron previamente codificados mediante un cifrado de siete números, no teniendo acceso a los datos clínicos durante el proceso de visualización de imágenes.

Los datos demográficos y clínicos fueron obtenidos, tras revisión de la historia clínica de los pacientes, en la anamnesis de la primera consulta de Neumología realizada dentro del protocolo de vigilancia postocupacional al asbesto, una vez los pacientes habían sido remitidos desde Atención Primaria. Se registraron el sexo y la edad, clasificándolos según su hábito tabáquico en?:

- Fumador activo: Persona que ha fumado al menos un cigarrillo al día en los últimos 6 meses.

- Ex-Fumador: Persona que, siendo fumadora, se ha mantenido sin fumar por lo menos los últimos 6 meses.

- No Fumador: Persona que nunca ha fumado o que fumó menos de 100 cigarrillos en su vida.

Se recuperó el grado de tabaquismo, registrado en paquetesaño ${ }^{8}$. A tal efecto, se consideró un cigarro puro equivalente a cinco cigarrillos.

Para los cálculos estadísticos se ha empleado el paquete estadístico SPSS en su versión IBM SPSS Statistics 19.0.0. Se estudia la asociacion entre variables cualitativas con el test chi-cuadrado. Se realiza la comparacion de medias con el test T de Student, tras comprobar la normalidad con el test de Kolmogorov-Smirnov, y la igualdad de varianzas con el test de Levene.

Para la participación en el estudio, se solicitó consentimiento informado escrito siguiendo las recomendaciones previamente realizadas por parte del Comité de Ética en Investigación Científica (CEIC) de Galicia, que con fecha 7 de Junio de 2012 autorizó la realización del presente trabajo (Código de registro 2012/136).

\section{Resultados}

El número total de pacientes incluidos en el estudio fue 55, y todos fueron de sexo masculino, con una edad media a la incorporación del estudio de 69,54 años. 
De los 55 pacientes, 20 (el 36,36\%) se declaraban no fumadores, 28 pacientes (el 50,91\%) se catalogaron como exfumadores en el momento de acudir a consulta, y 7 pacientes (el 12'73\%) como fumadores.

De los pacientes fumadores y ex-fumadores, se calculó el tabaquismo acumulado según los datos referidos en sus historias digitales, obteniendo un tabaquismo medio calculado en paquetes/año de 36,02 paquetes/año, con una desviación típica de 27,39 y una mediana de 30 paquetes/año. El tabaquismo acumulado mínimo registrado fue de 0'75 paquetes/ año, y el máximo, de 100 paquetes/año.

Al visualizar sus estudios de imagen, de los 55 pacientes, 10 presentaban datos de enfisema paraseptal (el 18,18\%) (Figura 1), 2 pacientes (el 3,64\%) enfisema centrolobulillar, y ningún paciente fue descrito como enfisema difuso.

Se identificó una diferencia estadísticamente significativa (Figura 2) al comparar los datos de tabaquismo acumulado en número de paquetes/año $(p=0,006)$ entre los pacientes que presentaban enfisema paraseptal y los que no, siendo la media de paquetes/año de los pacientes sin enfisema de 28,19 (desviación típica 23,25 paquetes/año) alcanzando dicha media en aquellos pacientes enfisematosos los 55,60 paquetes/año (con una desviación típica de 28,25 paquetes/año). No se encontraron diferencias estadísticas en el enfisema centrolobulillar, probablemente debido al escaso tamaño de la muestra. En la tabla 1 se adjuntan los datos poblacionales segregados en función de la presencia o no de enfisema paraseptal.

Tabla 1. Datos poblacionales

\begin{tabular}{|c|c|c|}
\hline & $\begin{array}{c}\text { No enfisema } \\
\text { paraseptal }\end{array}$ & $\begin{array}{c}\text { Enfisema } \\
\text { paraseptal }\end{array}$ \\
\hline Edad (años), media (DE) & $68,42(8,29)$ & $67,6(4,81)$ \\
\hline Hábito tabáquico (\%) & 8,89 & 30 \\
-Fumador activo & 46,67 & 70 \\
-Ex - fumador & 44,44 & 0 \\
-No fumador & & $55,60(28,25)$ \\
\hline $\begin{array}{c}\text { Tabaquismo acumulado } \\
\text { (paquetes -año), media (DE) }\end{array}$ & $28,19(23,25)$ & \\
\hline
\end{tabular}

\section{Discusión}

Que tanto el tabaco ${ }^{1}$ como la exposición previa a fibras de amianto ${ }^{3}$ inducen patología es algo conocido desde hace tiempo, al igual que su potenciación mutua ${ }^{4,5}$. Sin embargo, y pese a la elevada exposición que en determinadas regiones se tuvo a este tipo de sustancias (en nuestra área sanitaria, condicionada por la industria de construcción naval), no existen apenas artículos científicos publicados a este respecto. De hecho este es, en nuestro conocimiento, el primer artículo asociando tabaco y exposición al amianto con la aparición de enfisema en pacientes en España.

El tamaño muestral de este estudio fue de 55 pacientes, lo que constituye una cohorte relativamente pequeña si comparamos con otros estudios similares de valoración de enfisema en pacientes expuestos al amianto ${ }^{9}$, pero que es similar a otros estudios en este tipo de exposición al asbesto ${ }^{10}$, probablemente debido a la dificultad para reclutar pacientes dados los elevados períodos de latencia.

Del mismo modo, tampoco es infrecuente que todos los pacientes sean, como en el caso actual, de sexo masculino, pues en la práctica totalidad de estudios en pacientes expuestos al amianto el grupo de pacientes de sexo masculino es mayoritario -cuando no el único investigado-, tanto en los de comparación de pruebas ${ }^{10}$ como en aquellos estudios descriptivos poblacionales ${ }^{11}$. La edad media a la adquisición de las imágenes fue de 69,54 años, de igual manera similar a la de otros estudios en poblaciones expuestas al amianto de origen laboral ${ }^{12,13}$, aunque otros informes en poblaciones semejantes presentan una media de edad más joven ${ }^{14,15}$, habitualmente porque se trata de programas de cribado para cáncer de pulmón con criterios selectivos que excluyen a la población de más edad.

El grado de tabaquismo acumulado ha sido medido en paquetes-año siguiendo las recomendaciones de las sociedades médicas oficiales ${ }^{8}$, que en nuestros pacientes se encuentra en los 27,39 paquetes-año de media, dentro del rango habitual para este tipo de estudios, como refiere Wilken en su metaanálisis y revisión sistemática de trabajos publicados en trabajadores expuestos al amianto del año $2011^{16}$, con cifras que oscilan entre los 10,9 y los 38,9 paquetes-año.

Tabla 2. Grado de tabaquismo en España (Fuente: Instituto Nacional de Estadística -modificado por Macía-Suárez et al.-)

\begin{tabular}{|c|c|c|c|c|}
\hline & Fumador diario & Fumador ocasional & Ex-fumador & No fumador \\
\hline Varones 65-74 años & $16,16 \%$ & $1,76 \%$ & $20,26 \%$ & $31,82 \%$ \\
\hline Población general & $23,95 \%$ & $3,01 \%$ & $19,58 \%$ & $53,46 \%$ \\
\hline Población de estudio actual. & \multicolumn{2}{|c|}{$12,73 \%$} & $50,91 \%$ & $36,36 \%$ \\
\hline
\end{tabular}



asociando una pequeña banda parenquimatosa (flecha)

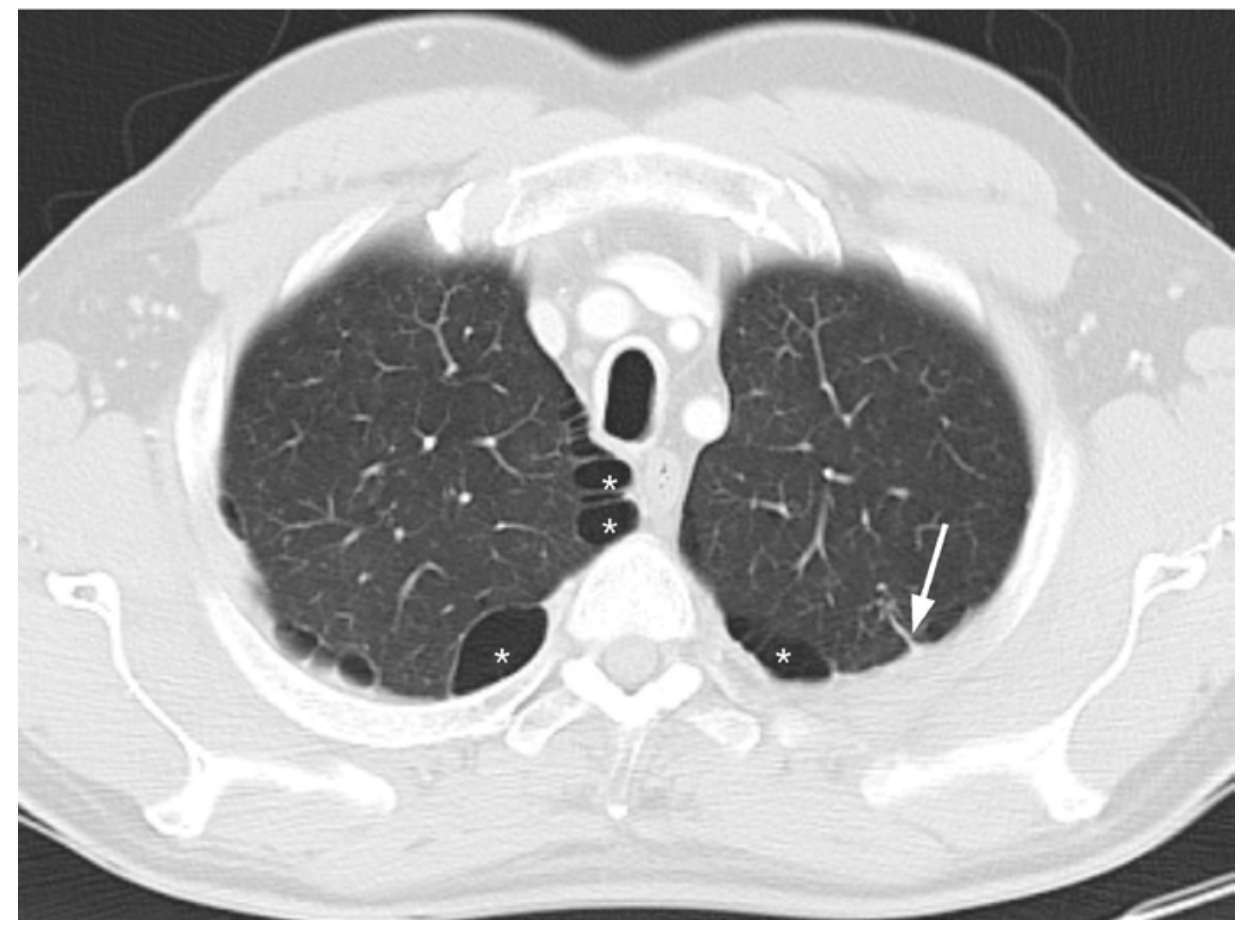

Si bien el porcentaje de tabaquismo activo de los pacientes a estudio es elevado (con un $36,36 \%$ de pacientes no fumadores, un $50,91 \%$ de ex-fumadores y un $12,73 \%$ de fumadores activos, Tabla 2) resulta igualmente equiparable al de poblaciones similares en estudios de cribado ${ }^{14,15}$ de patología secundaria a la exposición a asbesto, aunque en otras cohortes de pacientes, como en el caso del GB Asbestos Survey ${ }^{11}$, un estudio de cohortes prospectivo en trabajadores expuestos al amianto de ámbito nacional británico realizado desde 1971, el porcentaje de pacientes fumadores es mucho más elevado respecto al de ex-fumadores (58\% y $17 \%$ respectivamente), siendo semejante sin embargo el porcentaje de no fumadores (25\%). Este hecho puede deberse al carácter reglamentario que tiene ese protocolo (en lugar del carácter voluntario de otros programas como el nuestro), o a la inclusión en el mismo de trabajadores en activo, de menor edad y con mayor índice de tabaquismo activo ${ }^{17}$. En todo caso la proporción de fumadores es equiparable a la de la población de entre 65 y 75 años de edad (Tabla 2), con cifras mucho más elevadas de ex-fumadores que de fumadores respecto a la población general de España en el período 2011-2012 ${ }^{18}$. Comparando nuestros resultados, con un 18,18\% de pacientes con presencia de enfisema paraseptal (Figura 1) y un 3,64 $\%$ de pacientes con enfisema centrolobulillar, con el estudio de Remy-Jardin ${ }^{10}$ y el de Vierikko ${ }^{19}$, con cifras globales de enfisema (no diferencian entre tipos de enfisema) del 31\% y $22,6 \%$ respectivamente, y con el estudio de Huuskonen ${ }^{9}$, con ratios de enfisema moderado en el $29 \%$ de pacientes, y severo en un $5 \%$ adicional, nuestro porcentaje de pacientes enfisematosos es relativamente menor. Sin embargo, com- parativamente con el estudio de Vierikko ${ }^{19}$ existe una ratio de pacientes no fumadores bastante menor (el 36,36 \% en nuestro caso, respecto al $23,8 \%$ en el estudio de Vierikko), y en el de Remy-Jardin ${ }^{10}$ existe un índice de tabaquismo activo (31,33\% de fumadores, $36,14 \%$ de exfumadores) mucho mayor que en la cohorte de pacientes del presente trabajo. Con respecto al estudio de Huuskonen ${ }^{9}$ existen diferencias metodológicas que pueden justificar esta diferencia pues en él solo se seleccionaron pacientes diagnosticados de asbestosis. Pese al relativo tamaño de la muestra, se ha encontrado una diferencia estadísticamente significativa al comparar los datos de tabaquismo acumulado entre los pacientes que presentan enfisema paraseptal, siendo la media de paquetes/año de los pacientes sin enfisema de 28,19 paquetes/año frente a los 55,60 paquetes/año que alcanza en los pacientes con enfisema paraseptal. Este hecho estaría acorde con lo informado en estudios previos tanto en la población general ${ }^{20}$, como en estudios realizados en pacientes expuestos al amianto como el de Vehmas del año $2003^{4}$, que en un estudio realizado a 587 trabajadores de la construcción expuestos a asbesto, con TC espiral y TCAR, encontró que el tabaquismo incrementaba todos los datos de enfisema. Otros autores como Huuskonen ${ }^{9}$ han encontrado asociaciones entre la presencia de enfisema y de asbestosis establecida, pero nuestra muestra probablemente resulte demasiado pequeña para poder valorar una asociación similar.

En todo caso, cabe recordar lo ya expuesto en la introducción, sobre la relación multiplicativa (sinérgica) 0, al menos, más que sumatoria de los efectos nocivos del tabaco y el amianto en el parénquima pulmonar. Además del informe de Vehmas ${ }^{4}$, 
numerosos estudios han demostrado ${ }^{5}$ una asociación entre ambos factores y la aparición de patología, incluyendo la presencia de neoplasias ${ }^{21}$. Esta potenciación de efectos podría explicar que, pese al relativamente escaso tamaño muestral, hayamos podido identificar esta diferencia con significación estadística suficiente (Figura 2).

Figura 2. Representación gráfica de la comparación de medias del tabaquismo acumulado medido en paquetes/año entre pacientes sin enfisema paraseptal y con él

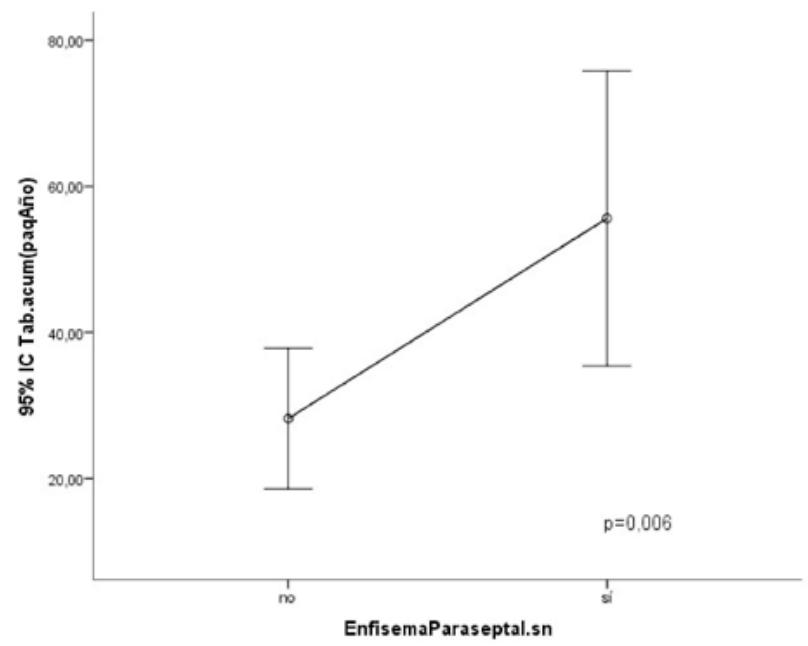

Durante la realización de este estudio se detectaron únicamente dos pacientes con enfisema centrolobulillar lo que impide realizar comparaciones estadísticamente significativas. La principal limitación de nuestro estudio radica en el tipo de selección de la muestra de pacientes. Más allá del carácter voluntario de su participación en el protocolo de seguimiento por Atención Primaria, hecho que probablemente minusvalora a los pacientes asintomáticos, únicamente aquellos pacientes que presentan algún tipo de alteración en el estudio rutinario son remitidos al centro de referencia para una evaluación más completa, lo que potencialmente puede originar que la población estudiada sea aquella con más afectación pulmonar. El pequeño tamaño muestral constituye otra de las limitaciones del estudio. Sin embargo, ha de tenerse en cuenta la dificultad para realizar un seguimiento a largo plazo de este tipo de pacientes, dados los largos períodos de latencia de este tipo de patología. El hecho, descrito por otros autores ${ }^{4,5,21}$, de la patogenia combinada entre el amianto y el tabaquismo, unido al despistaje previo realizado por medicina de Atención Primaria, justifican tal vez que podamos demostrar la relación entre el grado de tabaquismo y la presencia de enfisema paraseptal pese al relativo pequeño tamaño muestral.

En conclusión, dentro de los pacientes expuestos al amianto, aquellos con enfisema paraseptal muestran una tasa de tabaquismo acumulado superior a aquellos sin enfisema.

\section{Bibliografía}

1. Aj A. Cigarette smoking: health effects and control strategies. Drugs Today (Barc). 2008:44:895-904

2. Pernas Gómez M, Arencibia Flores L. Efectos sobre la salud de la exposición crónica al humo del tabaco en fumadores y no fumadores. Rev Cubana Med Gen Integral. 1998;14:180-4.

3. Vicente Pardo JM. Enfermedades respiratorias por exposición a amianto, aspectos clínico-laborales y médico-legales. Med Segur Trab. 2014;60:508-26.

4. Vehmas T, Kivisaari L, Huuskonen MS, Jaakkola MS. Effects of tobacco smoking on findings in chest computed tomography among asbestos-exposed workers. Eur Respir J. 2003;21:866-71.

5. Erren TC, Jacobsen M, Piekarski C. Synergy between Asbestos and Smoking on Lung: Cancer Risks. Epidemiology. 1999:10:405-11.

6. Webb R, Müller NL, Naidich DP, Valls Durán C. Alta resolución en TC de pulmón. 3a ed. Madrid: Marbán; 2003

7. Global adult tobacco survey collaborative group. Tobacco questions for surveys. A Subset of key questions from the Global Adult Tobacco Survey (GATS). 2Nd Edition. Atlanta, GA: Centers for disease control and prevention; 2011.

8. Jiménez Ruiz CA, Barrueco Ferrero M, Solano Reina S, Torrecilla García M, Domínguez Grandal F, Díaz-Maroto Muñoz JL, et al. Recomendaciones en el abordaje diagnóstico y terapéutico del tabaquismo . Documento de consenso. Arch Bronconeumol. 2003;39:35-41.

9. Huuskonen O, Kivisaari L, Zitting A, Kaleva S, Vehmas T. Emphysema Findings Associated with Heavy Asbestos-Exposure in High Resolution Computed Tomography of Finnish Construction Workers. J Occup Health. 2004;46:266-71.

10. Remy-Jardin M, Sobaszek A, Duhamel A, Mastora I, Zanetti C, Remy J. Asbestosrelated pleuropulmonary diseases: evaluation with low-dose four-detector row spiral CT. Radiology. 2004:233:182-90.

11. Frost $G$. The latency period of mesothelioma among a cohort of British asbestos workers (1978-2005). Br J Cancer. 2013;109:1965-73.

12. Fujimoto N, Kato K, Usami I, Sakai F, Tokuyama T, Hayashi S, et al. Asbestos-Related Diffuse Pleural Thickening. Respiration. 2014;88:277-84.

13. Gamsu G, Salmon CJ, Warnock ML, Blanc PD. CT quantification of interstitial fibrosis in patients with asbestosis: a comparison of two methods. Am J Roentgenol. 1995; 164:63-8.

14. Mastrangelo G, Ballarin MN, Bellini E, Bizzotto R, Zannol F, Gioffrè F, et al. Feasibility of a screening programme for lung cancer in former asbestos workers. Occup Med (Lond.) 2008:58:175-80.

15. Fasola G, Belvedere O, Aita M, Zanin T, Follador A, Cassetti P, et al. Low-dose computed tomography screening for lung cancer and pleural mesothelioma in an asbestosexposed population: baseline results of a prospective, nonrandomized feasibility tria an Alpe-adria Thoracic Oncology Multidisciplinary Group Study (ATOM 002). The Oncologist. 2007;12:1215-24.

16. Wilken D, Velasco Garrido M, Manuwald U, Baur X. Lung function in asbestos-exposed workers, a systematic review and meta-analysis. J Occup Med Toxicol. 2011; 6:21.

17. Jiménez Ruiz CA, Fernando Masa J, Sobradillo V, Gabriel R, Miravitlles M, FernándezFau $L$, et al. Prevalencia y actitudes sobre tabaquismo en población mayor de 40 años. Arch Bronconeumol. 2000;36:241-4.

18. Instituto Nacional de Estadística. (National Statistics Institute) [Internet]. [citado $30 \mathrm{de}$ enero de 2017]. Disponible en: http://www.ine.es/jaxi/Tabla.htm?path=/t15/p419/ a2006/p03/l0/\&file=03001.px\&L=0

19. Vierikko T, Järvenpää R, Toivio $P$, Uitti J, Oksa $P$, Lindholm T, et al. Clinical and HRCT screening of heavily asbestos-exposed workers. Int Arch Occup Environ Health. 2010;83:47-54.

20. Spaggiari E, Zompatori M, Verduri $A$, Chetta $A, B$ à C, Ormitti F, et al. Early smokinginduced lung lesions in asymptomatic subjects. Correlations between high resolution dynamic CT and pulmonary function testing. Radiol Med (Torino). 2005;109:27-39.

21. Balmes JR. Asbestos and lung cancer: what we know. Am J Respir Crit Care Med. 2013:188:8-9. 